\title{
Properties of Surface Modes Used for Directional Emission from Photonic Crystal Waveguides
}

\author{
K. B. Chung* \\ School of Electronic and Electrical Engineering, Hongik University, Seoul 121-791, Korea
}

(Received December 3, 2007 : revised January 4, 2008)

\begin{abstract}
Directional emission of light exiting a photonic crystal waveguide by a coherent action of radiative surface modes was recently demonstrated, and subsequently the substantial enhancement of the directional emission was achieved by engineering the surface and adjusting relevant parameters. Here we present the analysis of surface modes causing the enhanced emission by the plane wave expansion method and the finite-difference time-domain method. In particular, surface band structures are calculated for nonradiative and radiative surface modes, respectively, and intensity profiles of some representative modes for nonradiative and radiative cases are given.

OCIS codes : $050.0050,240.6690$
\end{abstract}

\section{INTRODUCTION}

Surface waves on finite photonic crystals (PCs) [1] are electromagnetic waves localized at the interface between a PC and another medium, similar to surface waves on metallic surfaces or crystalline solids. Initially, it was argued that any surface of any PCs with a band gap would always have surface states for some termination, but surface waves in PCs were regarded as an undesirable feature that would cause radiation loss in semiconductor lasers or LEDs [1]. Most theoretical and experimental works that have followed the original proposal have focused on the existence and physics of surface waves [2-7]. Recently, for metallic films, beaming phenomenon from a single subwavelength aperture surrounded by a periodic corrugation on a film was reported $[8,9]$. When similar effect was observed also in PCs $[10,11]$, interest in surface waves in PCs arose again. While surface waves on metallic surfaces are due to surface plasmons at an optical frequency, surface waves on PC surfaces are intentionally generated by surface modification or termination. By applying the basic concept used for metallic films, beaming or directional emission exiting from a PC waveguide less than a wavelength wide was theoretically [10] and experimentally [11] demonstrated, followed by a number of reports [12-16]. Very recently, a quantitative model explaining the physical mechanism of the directional emission was reported [16]. It is believed that the beaming by surface waves in PCs would find applications in nano-focusing and near-field optical devices. It may be helpful also in the coupling of $\mathrm{PC}$ waveguides with conventional photonic devices, such as dielectric waveguides and optical fibers.

The directional emission from two-dimensional PC waveguides proposed originally in Ref. [10] was later enhanced by engineering the surface and adjusting relevant parameters $[14,15]$. These modifications include using a positive surface corrugation, increasing the refractive index of the surface layer, inducing a nearsurface defect mode, and adjusting the input wavelength in consequence [14]. Although Ref. [14] demonstrated the enhancement of directional emission by numerical simulation, the behavior of the surface modes accountable for the enhancement and the physics behind the chosen values of relevant parameters were not reported. In this paper, we present the analysis of surface modes causing the enhanced emission by the plane wave expansion (PWE) method $[17,18]$ and the finitedifference time-domain (FDTD) method [19-21]. In particular, surface band structures are calculated for nonradiative and radiative surface modes, respectively, and intensity profiles of some representative modes for nonradiative and radiative cases are given. The nonradiative surface modes are normal surface waves localized at and propagating along an interface between two media, whereas the radiative surface modes are surface waves that radiate away from the interface during propagation along the interface. The radiative modes are usually obtained by adding surface corrugation, whose physics is explained below. 


\section{MODEL AND METHODS OF COMPUTATION}

The photonic crystal structure that we study here is shown in Fig. 1 (a). Its basic structure is adopted from Refs. [10] and [14]. The underlying PC is a two-dimensional square lattice of dielectric cylinders in air $\left(\mathcal{E}_{r}=\right.$ 11.56 and $r / a=0.18$, where $r$ is the radius of cylinders and $a$ is the lattice constant.). We consider TM polarization (the electric field parallel to the axis of the cylinders), for which the PC has a band gap from $\omega a / 2 \pi c=0.30$ to $\omega a / 2 \pi c=0.44$ ( $c$ is the speed of light.). At the center of the structure, a waveguide is formed by eliminating a line of cylinders. The radii of the surface cylinders at the PC-air interface are reduced to $r_{s} / a=0.09$ to induce surface modes [10], which are nonradiative modes localized at the interface. To obtain directional emission by a coherent action of radiative surface modes, surface corrugation with displacement $\Delta \mathrm{z}$ of odd-numbered surface cylinders (numbered to both sides from the waveguide exit) and with period $\Lambda$ is created at the interface. This corrugation moves surface mode momentum, by adding its corrugation momentum, into the light cone [1], in which propagating modes in air reside in a dispersion

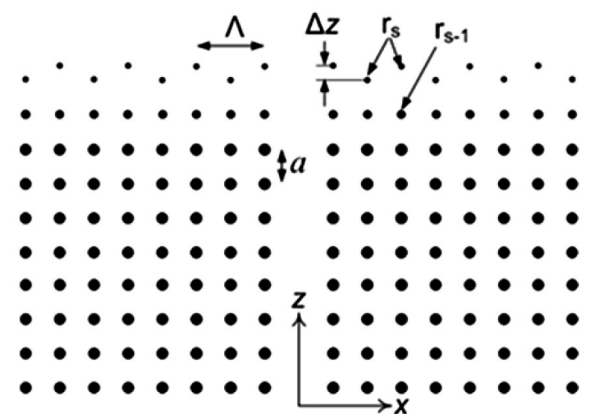

(a)

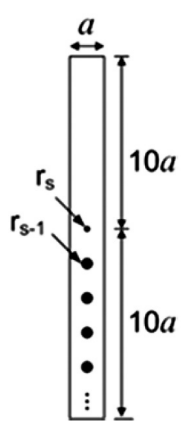

(b)

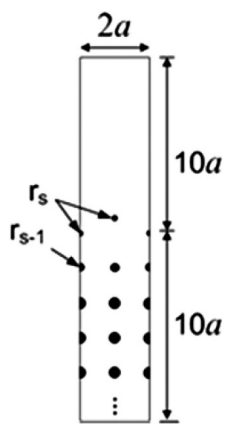

(c)
FIG. 1. (a) Photonic crystal structure for directional emission. (b) Supercell for the calculation of surface band structures of nonradiative surface modes for $\Delta \mathrm{z}=0$ and $r_{s-1}=r=0.18 a$ by the PWE method. (c) Supercell for the calculation of surface band structures of radiative surface modes by the FDTD method with periodic boundary conditions. diagram. Emission by radiative surface modes could also be understood by considering that the displaced cylinders by the corrugation act as scattering centers for surface modes. In Fig. 1 (a), $r_{s-1}$ is the radius of the cylinders next to the surface cylinders.

To obtain surface band structures or dispersion diagrams for surface modes, one usually applies the PWE method to a supercell, a widely-used method in calculating band structures $[17,18]$ and surface band structures $[1-3,6]$. Here we also use the PWE method to compute the surface band structures for nonradiative surface modes. The supercell we adopt is drawn in Fig. 1 (b). Its size is a and $20 a$ along the $x$ and $\mathrm{z}$ directions, respectively. Its air region is sufficiently large to avoid spurious modes that originate from the coupling of modes with those in nearby supercells. The supercells are implicitly assumed to repeat indefinitely in both directions because of periodicity assumption inherent in the PWE method.

For surface band structures for radiative surface modes, however, we need to adopt the supercell shown in Fig. 1 (c) with its size being $2 a$ and $20 a$, due to the displacement $\Delta z$ of every other surface cylinder. When one applies the PWE method to the supercell, band folding occurs in a band structure, because the Brillouin zone is reduced to a half in the $x$ direction. As a result, it is nearly impossible to distinguish true modes from spurious modes induced from band folding. Here we use the FDTD method [19-21] with periodic boundary conditions $[22,23]$ to compute surface band structures $[4,15]$ for radiative surface modes. We apply the periodic boundary condition and the perfectly matched layer [20] at the boundaries in the $x$ and $\mathrm{z}$ directions, respectively. In the supercell in Fig. 1 (c), we place two current point sources with impulse response in time near the interface. The two sources separated by a lattice vector of the square lattice are needed in order to obtain unfolded surface band structures $[15,23]$. The relative phase between the sources is given by Bloch's theorem. To observe the field, we place several monitors at the interface and in the PC bulk and air regions. For a fixed value of surface wave vector, the monitors store field data as a function of time, while the FDTD time marching proceeds. After the single FDTD run is completed, the field value at each monitor is transformed into the frequency domain by the fast Fourier transform algorithm, and is added up to a single spectral data in which peaks are found by a peak search algorithm. The peaks correspond to the eigenfrequencies of surface modes, PC bulk modes, and air modes residing in the light cone, for a single value of surface wave vector.

Intensity profiles of surface modes are computed by the PWE method with the same supercells used in calculating the surface band structures, because it is easier and faster in obtaining mode profiles. For nonradiative surface modes, mode intensity profiles are computed easily in the framework of the PWE method by giving the frequency and wave vector of a desired mode, 
which are usually obtained from the surface band structure. For radiative surface modes, the surface band structures are calculated by the FDTD method, as described previously. After the frequency and wave vector of a desired mode are chosen from the band structure, mode intensity profiles are computed similarly by the PWE method. Because of band folding that occurs for the supercell in Fig. 1 (c), there appear many spurious modes with similar frequencies and wave vectors. So care was taken in selecting the mode profiles of desired modes.

\section{RESULTS AND DISCUSSION}

First, the surface band structures for nonradiative surface modes are presented in Fig. 2. The band structure is a plot of surface mode frequency versus surface wave vector. In the following figures, the gray areas in the upper and lower parts are PC bulk bands, and the area with varying shade is the light cone in which propagating modes in air reside. The dark areas are

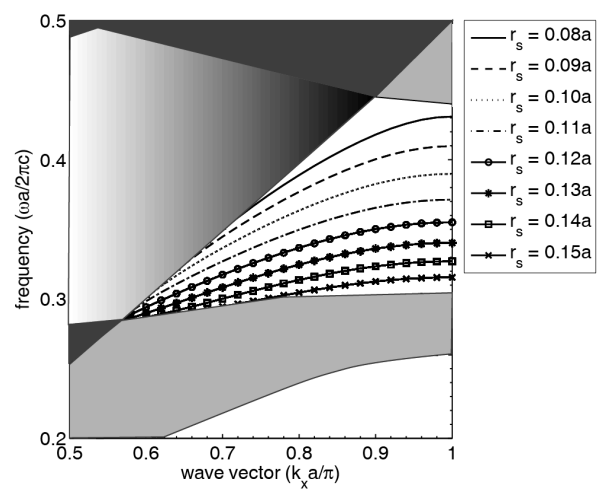

(a)

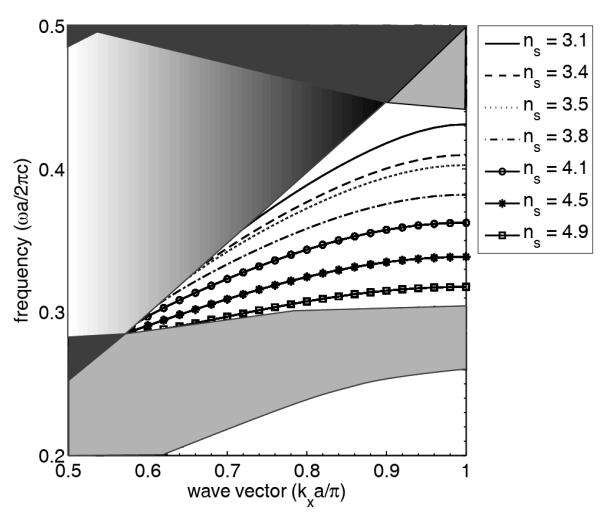

(b)

FIG. 2. Surface band structures for nonradiative surface modes with $\Delta \mathrm{z}=0$ and $r_{s-1}=0.18 a$ for the surface cylinders (a) with different $r_{s}$ and $n_{s}=3.4$, and (b) with different $n_{s}$ and $r_{s}=0.09 a$. overlaps of the PC bulk band and the light cone. Surface modes exist in the common area that belongs to a band gap and is outside the light cone. As a result, they decay both in the bulk PC and in air, and are localized at the interface. Figure 2 (a) shows surface mode frequency for different radii $r_{s}$ with a fixed refractive index $n_{s}=3.4$ (the index value of the cylinders in the bulk PC) of surface cylinders. We see that the mode frequency is sensitive to variation of $r_{s}$ and spans the almost entire range of the band gap with different $r_{s}$. For different refractive index $n_{s}$, surface mode frequency is shown in Fig. 2 (b) with a fixed radius $r_{s}$ $=0.09 \mathrm{a}$. The sensitivity to $n_{s}$ and coverage of almost the entire band gap are also observed.

In Fig. 3, the intensity profiles of some representative nonradiative surface modes for no displacement of surface cylinders are shown. Figure 3 (a) clearly demonstrates the existence of a surface mode that is localized at the interface and does not propagate either into the bulk PC or into air. For a larger radius of surface cylinders, the mode size is bigger with a smaller intensity peak, as shown in Fig. 3 (b). For a smaller

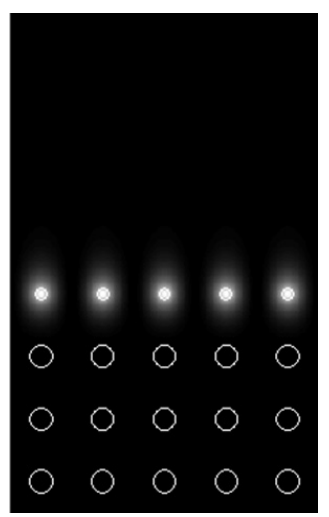

(a)

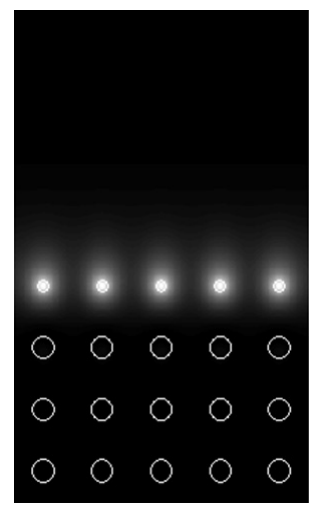

(c)

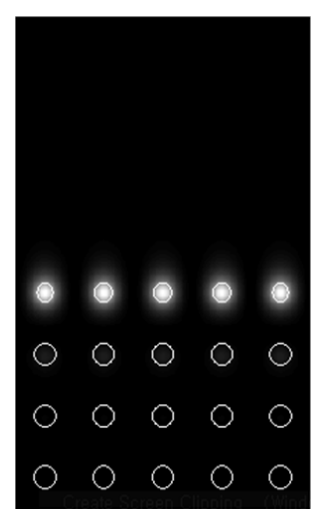

(b)

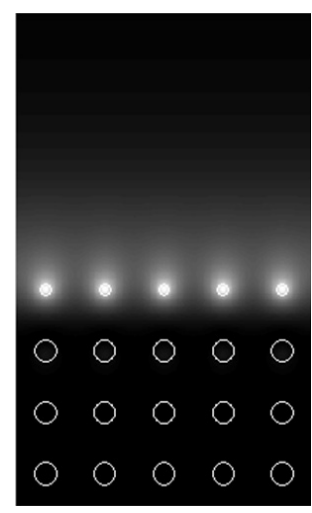

(d)
FIG. 3. Intensity profiles of nonradiative surface modes for $\Delta \mathrm{z}=0, r_{s-1}=0.18 \mathrm{a}$, and $n_{s}=3.4$. (a) $r_{s}=0.09 \mathrm{a}$ and $k_{x} a / \pi$ $=1.0$. (b) $r_{s}=0.15 a$ and $k_{x} a / \pi=1.0$. (c) $r_{s}=0.09 a$ and $k_{x} a / \pi=0.8$. (d) $r_{s}=0.09 a$ and $k_{x} a / \pi=0.7$. 
wave vector $k_{x} a / \pi=0.8$, with the same $r_{s}=0.09 a$ as in Fig. 3 (a), this mode is less localized at the interface and spreads somewhat into air in Fig. 3 (c), because the mode is close to the light line. In Fig. 3 (d), the mode is very close to the light line with $k_{x} a / \pi=0.7$, so it spreads significantly into air, as seen similarly in Fig. 1 of Ref. [24].

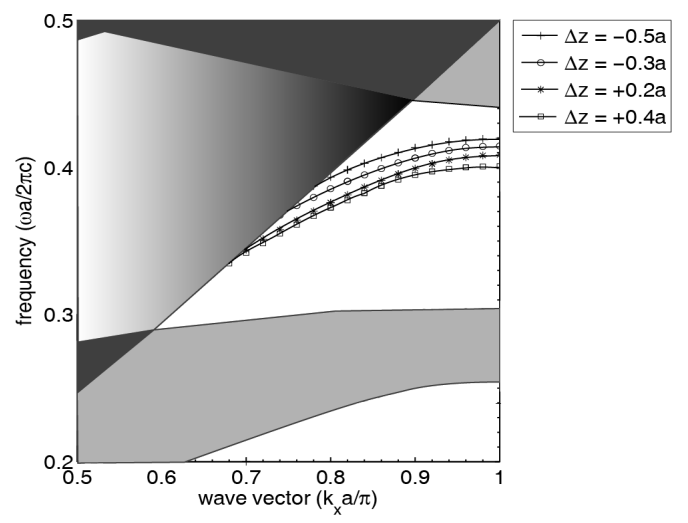

(a)

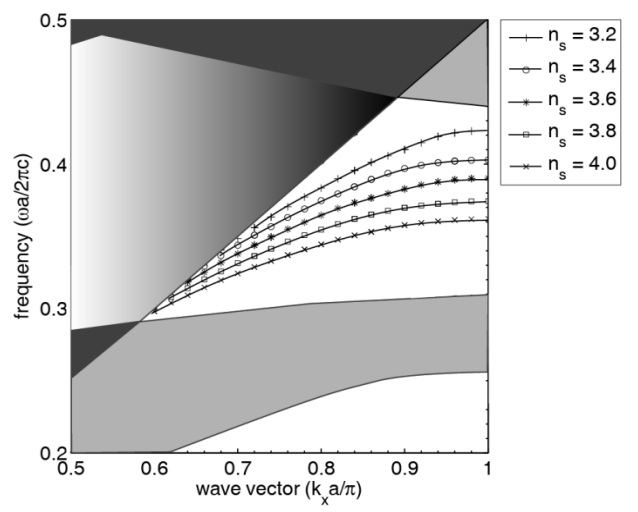

(b)

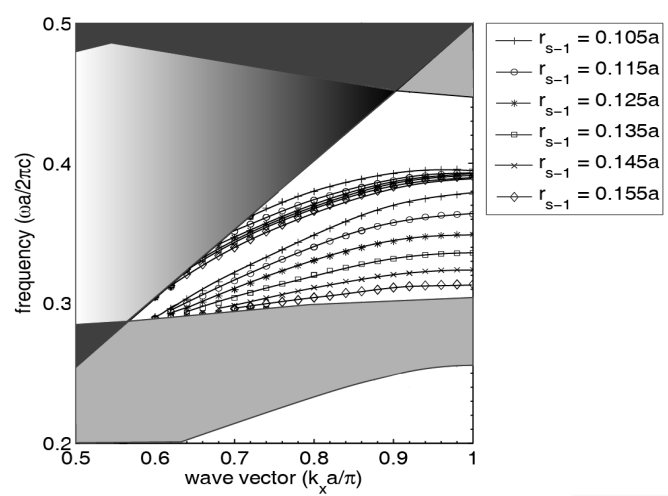

(c)

FIG. 4. Surface band structures for radiative surface modes (a) for different $\Delta \mathrm{z}$ with $r_{s}=0.09 a, n_{s}=3.4$, and $r_{s-1}=0.18 a(\mathrm{~b})$ for different $n_{s}$ with $\Delta \mathrm{z}=+0.4 a, r_{s}=$ $0.09 a$, and $r_{s-1}=0.18 a$, and (c) for different $r_{s-1}$ with $\Delta \mathrm{z}$ $=+0.4 a, n_{s}=3.6$, and $r_{s}=0.09 a$.
Next, in Fig. 4, the surface band structures for radiative surface modes are presented for variations of three respective parameters $\Delta \mathrm{z}, n_{s}$, and $r_{s-1}$. Figure 4 (a) shows surface mode frequency for different surface displacement $\Delta \mathrm{z}$, except which the other parameters are similar to those in the nonradiative case in Fig. 2. The case with $\Delta \mathrm{z}=-0.3 a$ corresponds to the original proposal reported in Ref. [10]. We see a blue shift of the mode frequency for negative displacement and a red shift for positive displacement, even though it does not vary much for different displacement. The variation of the mode frequency for different refractive index $n_{s}$ with a fixed $\Delta \mathrm{z}=+0.4 a$ is given Fig. 4 (b). The mode frequency is relatively sensitive to $n_{s}$ and decreases with an increasing $n_{s}$. For different radii $r_{s-1}$ of near-surface cylinders, it is shown in Fig. 4 (c) with fixed values of $\Delta \mathrm{z}=+0.4 a, n_{s}=3.6$, and $r_{s}=0.09 a$. In addition to the surface modes seen similarly in the other figures, there appear near-surface defect modes with smaller frequencies for all cases with $r_{s-1}<r$. The case with $r_{s-1}=0.135 \mathrm{a}$ corresponds to that of the enhanced emission reported in Ref. [14]. The frequency of the nearsurface defect mode is sensitive to $r_{s-1}$ and decreases with an increasing $r_{s-1}$.

Figure 5 presents the intensity profiles of some representative radiative surface modes for $r_{s-1}=0.18 a$ and $r_{s}=0.09 a$. The mode in Fig. 5 (a) is the radiative surface mode reported in Ref. [10] for negative displacement $\Delta \mathrm{z}=-0.3 a$. The field intensity is strong on negatively displaced cylinders with some weak field on undisplaced cylinders. For positive displacement $\Delta \mathrm{z}=$ $+0.4 a$, the intensity profile is given in Fig. 5 (b), where most field is concentrated on positively displaced cylinders. We note that some weak field radiates into air because it is a radiative mode, although it is not seen clearly in the figure. Figure 5 (c) shows the intensity profile of a mode very close to the light line. Its field spreads significantly into air and is strong on the undisplaced cylinders. In Fig. 5 (d), the refractive index of the surface cylinders increases to $n_{s}=3.6$ from $n_{s}$ $=3.4$ as in Fig. 5 (b). The intensity profile looks quite similar, but the field radiation into air is a little more visible.

Finally, Fig. 6 shows the intensity profiles of two modes for $\Delta \mathrm{z}=+0.4 a, r_{s-1}=0.135 a, n_{s}=3.6$, and $r_{s}$ $=0.09 a$ at $k_{x} a / \pi=1.0$. Figure 6 (a) is that for a radiative surface mode. It is quite similar to Fig. 5 (d) with $r_{s-1}=0.18 \mathrm{a}$, and its field radiates into air. This mode is the radiative mode reported in Ref. [14], in which its mode pattern was not given. We give some explanation on this argument. According to Ref. [10], the corrugation period $\Lambda$ should coincide with a surface mode wavelength $\lambda$ to obtain beaming in the forward direction. Given $\Lambda=2 a$, the wave vector of surface modes is $k_{x}= \pm 2 \pi / \lambda= \pm \pi / a$, which is at the Brillouin zone edge. The wavelength used in Fig. 1 (c) in Ref. [14] is 


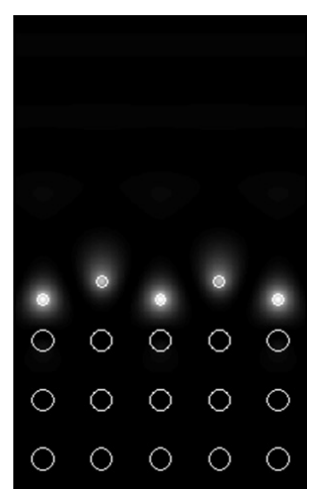

(a)

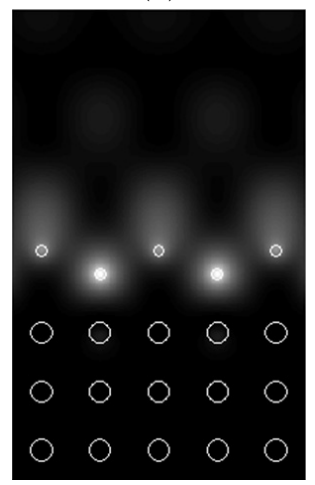

(c)

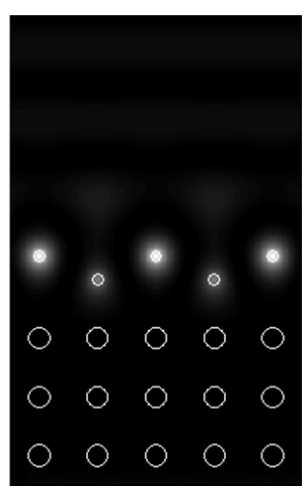

(b)

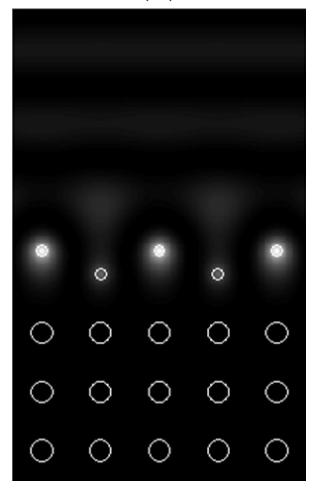

(d)
FIG. 5. Intensity profiles of radiative surface modes for $r_{s-1}=0.18 a$ and $r_{s}=0.09 a$. (a) $\Delta \mathrm{z}=-0.3 a, n_{s}=3.4$, and $k_{x} a / \pi=1.0$. (b) $\Delta \mathrm{z}=+0.4 a, n_{s}=3.4$, and $k_{x} a / \pi=1.0$. (c) $\Delta \mathrm{z}=+0.4 a, n_{s}=3.4$, and $k_{x} a / \pi=0.7$. (d) $\Delta \mathrm{z}=+0.4 a$, $n_{s}=3.6$, and $k_{x} a / \pi=1.0$.

$\lambda \sim 2.6386 \mathrm{a}$, which corresponds to $\omega a / 2 \pi c=a / \lambda=0.37899$. Although it is not clearly stated in Ref. [14], we believe this wavelength occurs at the Brillouin zone edge. This wavelength corresponds to the case $r_{s 1}=0.135 a$ at $k_{x} a / \pi$ $=1.0$ in Fig. 4 (c) despite a small numerical discrepancy. We can see clearly the difference between Fig. 5 (a) corresponding to Ref. [10] and Fig. 6 (a) corresponding to Ref. [14]. This difference may, at least qualitatively, explain the directional emission reported in Ref. [14] that is enhanced and optimized from that in Ref. [10]. Figure 6 (b) gives the intensity pattern of a near-surface defect mode that is localized on the nearsurface cylinder layer as expected. It will be published elsewhere how the radiative surface mode and the nearsurface defect mode interact and contribute to the enhanced directional emission, as described in Ref. [14].

\section{CONCLUSION}

In this paper, we have presented the analysis of surface modes causing the enhanced directional emission by the plane wave expansion method and the finite-difference time-domain method. In particular, surface band structures

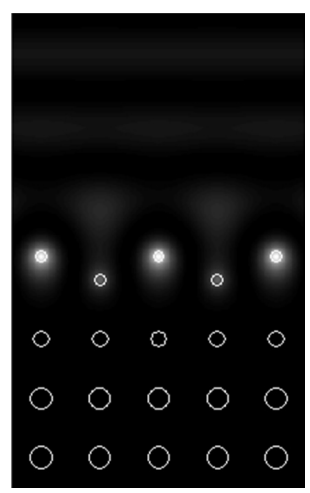

(a)

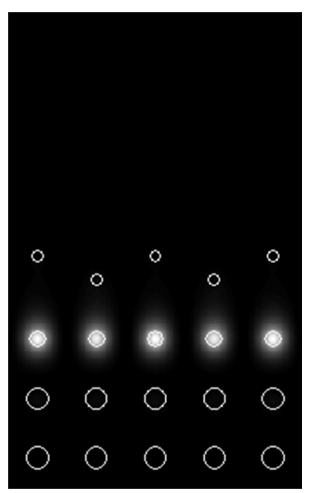

(b)
FIG. 6. Intensity profiles of (a) a radiative surface mode and (b) a near-surface defect mode, with $\Delta \mathrm{z}=+0.4 a, r_{s-1}$ $=0.135 a, n_{s}=3.6, r_{s}=0.09 a$, and $k_{x} a / \pi=1.0$.

for nonradiative surface modes for different radii and refractive index of surface cylinders were calculated. For radiative surface modes, surface band structures for different corrugation displacement, for different refractive index of surface cylinders, and for different radii of near-surface cylinders were calculated. The intensity profiles of some representative modes for the nonradiative case were given. For the radiative case, the intensity profiles of radiative surface modes causing the enhanced emission and of near-surface defect modes were given. We believe that the results presented here may be useful in designing and enhancing surface waveguides in photonic crystals [24-26].

\section{ACKNOWLEDGEMENT}

The author would like to acknowledge the comments by anonymous reviewers, which make the content of this paper clearer.

This work was supported by the 2007 Hongik University Research Fund.

*Corresponding author: kbchung@hongik.ac.kr

\section{REFERENCES}

[1] R. D. Meade, K. D. Brommer, A. M. Rappe, and J. D. Joannopoulos, "Electromagnetic Bloch waves at the surface of a photonic crystal," Phys. Rev. B, vol. 44, no. 19, pp. 10961-10964, 1991.

[2] W. M. Robertson, G. Arjavalingam, R. D. Meade, K. D. Brommer, A. M. Rappe, and J. D. Joannopoulos, "Observation of surface photons on periodic dielectric arrays," Opt. Lett., vol. 18, no. 7, pp. 528-530, 1993.

[3] F. Ramos-Mendieta and P. Halevi, "Surface electromagnetic waves in two-dimensional photonic crystals: Effect of the position of the surface plane," Phys. Rev. B, vol. 59, no. 23, pp. 15112-15120, 1999. 
[4] M. Qiu and S. He, "Surface modes in two-dimensional dielectric and metallic photonic band gap structures: a FDTD study," Phys. Lett. A, vol. 282, no. 1-2, pp. 8591, 2001.

[5] S. Enoch, E. Popov, and N. Bonod, "Analysis of the physical origin of surface modes on finite-size photonic crystals," Phys. Rev. B, vol. 72, no. 15, pp. 155101-1155101-7, 2005.

[6] B. Wang, W. Dai, A. Fang, L. Zhang, G. Tuttle, Th. Koschny, and C. M. Soukoulis, "Surface waves in photonic crystal slabs," Phys. Rev. B, vol. 74, no. 19, pp. 195104-1-195104-4, 2006.

[7] S. Foteinopoulou, M. Kafesaki, E. N. Economou, and C. M. Soukoulis, "Backward surface waves at photonic crystals," Phys. Rev. B, vol. 75, no. 24, pp. 2451161-245116-6, 2007.

[8] H. J. Lezec, A. Degiron, E. Devaux, R. A. Linke, L. Martin-Moreno, F. J. Garcia-Vidal, and T. W. Ebbesen, "Beaming light from a subwavelength aperture," Science, vol. 297, no. 5582, pp. 820-822, 2002.

[9] L. Martin-Moreno, F. J. Garcia-Vidal, H. J. Lezec, A. Degiron, and T. W. Ebbesen, "Theory of highly directional emission from a single subwavelength aperture surrounded by surface corrugations," Phys. Rev. Lett., vol. 90, no. 16, pp. 167401-1167401-4, 2003.

[10] E. Moreno, F. J. Garcia-Vidal, and L. Martin-Moreno, "Enhanced transmission and beaming of light via photonic crystal surface modes," Phys. Rev. B, vol. 69, no. 12, pp. 121402-1-121402-4, 2004.

[11] P. Kramper, M. Agio, C. M. Soukoulis, A. Birner, F. Muller, R. B. Wehrspohn, U. Gosele, and V. Sandoghdar, "Highly directional emission from photonic crystal waveguides of subwavelength width," Phys. Rev. Lett., vol. 92, no. 11, pp. 113903-1-113903-4, 2004.

[12] E. Moreno, L. Martin-Moreno, and F. J. Garcia-Vidal, "Efficient coupling of light into and out of a photonic crystal waveguide via surface modes," Photonics Nanostruc. - Fundamen. Appl., vol. 2, no. 2, pp. 97-102, 2004.

[13] I. Bulu, H. Caglayan, and E. Ozbay, "Beaming of light and enhanced transmission via surface modes of photonic crystals," Opt. Lett., vol. 30, no. 22, pp. 3078-3080, 2005.

[14] S. K. Morrison and Y. S. Kivshar, "Engineering of directional emission form photonic-crystal waveguides," Appl.
Phys. Lett., vol. 86, no. 8, pp. 081110-1-081110-3, 2005.

[15] S. K. Morrison and Y. S. Kivshar, "Beaming effect and directional emission from photonic-crystal waveguides," Proc. SPIE, vol. 5733, pp. 104-113, 2005.

[16] W. Smigaj, "Model of light collimation by photonic crystal surface modes," Phys. Rev. B, vol. 75, no. 20, pp. 205430-1-205430-8, 2007.

[17] K. M. Ho, C. T. Chan, and C. M. Soukoulis, "Existence of a photonic gap in periodic dielectric structures," Phys. Rev. Lett., vol. 65, no. 25, pp. 3152-3154, 1990.

[18] R. D. Meade, A. M. Rappe, K. D. Brommer, J. D. Joannopoulos, and O. L. Alerhand, "Accurate theoretical analysis of photonic band-gap materials," Phys. Rev. $B$, vol. 48, no. 11, pp. 8434-8437, 1993.

[19] K. S. Yee, "Numerical solution of initial boundary value problems involving Maxwell's equations in isotropic media," IEEE Trans. Antennas Propag., vol. AP-14, no. 3, pp. 32-307, 1966.

[20] J. P. Berenger, "A perfectly matched layer for the absorption of electromagnetic waves," J. Comput. Phys., vol. 114, no. 2, pp. 185-200, 1994.

[21] A. Taflove and S. C. Hagness, Computational Electrodynamics: The Finite-Difference Time-Domain Method (Third Edition, Artech House, Boston, U.S.A., 2005) Chapter 16.

[22] C. T. Chan, Q. L. Yu, and K. M. Ho, "Order-N spectral method for electromagnetic waves," Phys. Rev. B, vol. 51, no. 23, pp. 16635-16642, 1995.

[23] S. Fan, P. R. Villeneuve, and J. D. Joannopoulos, "Large omnidirectional band gaps in metallodielectric photonic crystals," Phys. Rev. B, vol. 54, no. 16, pp. 1124511251, 1996.

[24] A. I. Rahachou and I.V. Zozoulenko, "Waveguiding properties of surface states in photonic crystals," J. Opt. Soc. Am. B, vol. 23, no. 8, pp. 1679-1683, 2006.

[25] H. Chen, K. K. Tsia, and A. W. Poon, "Surface modes in two-dimensional photonic crystal slabs with a flat dielectric margin," Opt. Express, vol. 14, no. 16, pp. 7368-7377, 2006.

[26] E. H. Khoo, T. H. Cheng, A. Q. Liu, J. Li, and D. Pinjala, "Transmitting light efficiency on photonic crystal surface waveguide bend," Appl. Phys. Lett., vol. 91, no. 17, pp. 171109-1-171109-3, 2007. 